\title{
Comprehensive transcriptome analysis of early male and female Bactrocera jarvisi embryos
}

\author{
Jennifer L Morrow ${ }^{1 *}$, Markus Riegler ${ }^{1}$, A Stuart Gilchrist ${ }^{2}$, Deborah CA Shearman², Marianne Frommer ${ }^{2}$
}

\begin{abstract}
Background: Developing embryos are provided with maternal RNA transcripts and proteins, but transcription from the zygotic nuclei must be activated to control continuing embryonic development. Transcripts are generated at different stages of early development, and those involved in sex determination and cellularisation are some of the earliest to be activated. The male sex in tephritid fruit flies is determined by the presence of a $Y$ chromosome, and it is believed that a transcript from the $\mathrm{Y}$-chromosome sets in motion a cascade that determines male development, as part of the greater maternal to zygotic transition (MTZ). Here we investigate the poly $\left(\mathrm{A}^{+}\right)$ transcriptome in early male and female embryos of the horticultural pest Bactrocera jarvisi (Diptera: Tephritidae).

Results: Bactrocera jarvisi embryos were collected over two pre-blastoderm time periods, 2-3h and 3-5h after egg laying. Embryos were individually sexed using a Y-chromosome marker, allowing the sex-specific poly $\left(\mathrm{A}^{+}\right)$ transcriptome of single-sex embryo pools to be deep-sequenced and assembled de novo. Transcripts for sixteen sex-determination and two cellularisation gene homologues of Drosophila melanogaster (Diptera: Drosophilidae) were identified in early embryos of B. jarvisi, including transcripts highly upregulated prior to cellularisation. No strong candidates for transcripts derived solely from the $Y$ chromosome were recovered from the poly $(A+)$ fraction.

Conclusions: Bactrocera jarvisi provides an excellent model for embryonic studies due to available Y-chromosome markers and the compact time frame for zygotic transcription and the sex-determined state. Our data contribute fundamental information to sex-determination research, and provide candidates for the sourcing of gene promoters for transgenic pest-management strategies of tephritid fruit flies.
\end{abstract}

\section{Background}

Early stages of embryonic development involve large changes to the RNA transcript profile, as maternal transcripts, deposited during oogenesis, are targeted for degradation, and activation of the zygotic genome takes place. In Drosophila melanogaster, egg activation is triggered by osmotic and physical stimulation and occurs independently of fertilisation. Proteins such as SMAUG (SMG) and microRNAs are required to regulate degradation of maternal mRNAs in the developing embryo [1-3]. At least $30 \%$ of the transcripts in the D. melanogaster early embryo have the distinctive expression profile of maternal transcripts and about two thirds of these decrease markedly over the first $6.5 \mathrm{~h}$ of development [4].

\footnotetext{
* Correspondence: j.morrow@uws.edu.au

'Hawkesbury Institute for the Environment, University of Western Sydney, Locked Bag 1797, Penrith, NSW 2751, Australia

Full list of author information is available at the end of the article
}

During these early stages, transcription from the zygotic genome must be initiated. Activation of zygotic transcription is controlled, in part, by the zelda (zld) protein, which interacts with specific heptamer motifs (TAGteam sites) located in the regulatory regions upstream of genes targeted for early, pre-blastoderm transcription [5,6]. Some of the earliest genes to be transcribed are involved in sex determination, such as sisterless $A$ (sisA) during nuclear cycle 8 [7]. From cycle 11, cellularisation genes including serendipity $\alpha($ sry $\alpha)$, nullo, bottleneck (bnk) and slow as molasses (slam) are activated [8-11].

The genes of the sex-determination pathway are highly conserved in Diptera, most notably at the terminal gene, doublesex ( $d s x$ ) [12], and its upstream regulatory genes transformer (tra) [13] and transformer-2 (tra-2) [14]. In D. melanogaster, sex-specific splicing of tra, which generates the active TRA protein in females and a non-functional protein in males, is regulated by the Sex-lethal protein (SXL). Ongoing production of 
functional SXL occurs in females because an early, transiently-generated $S x l$ protein is produced only in females in response to the primary signal. The mRNA transcribed from the late promoter is spliced in the female-specific mode only when the early SXL is present. The primary signal that leads to female-specific $S x l$ is transmitted by a combination of X-chromosomelinked signal elements (XSE), namely sisA, scute (sc), outstretched (os) and runt (run), whose protein products are more concentrated in XX females than $\mathrm{XY}$ males [15]. These gene products interact with maternal products daughterless (da), hermaphrodite (her), extramacrochaetae (emc) and groucho (gro) and the zygotically-expressed autosomal gene deadpan (dpn) [reviewed in [16]]. Many of these genes have other molecular functions in development, and have been co-opted into a sex-determination regulatory role in Drosophila as the apex of the pathway diverged from other Diptera.

In contrast to Drosophila, Sxl transcripts are not sexspecifically spliced in other Diptera including tephritid species $[17,18]$ and house fly [19], and thus have no clear role in sex-determination. Homologues for some of the genes involved in regulation of $S x l$ in D. melanogaster have been identified in EST libraries of the tephritid fruit fly Ceratitis capitata [20]. Transcript expression analysis in unfertilised eggs and early developing embryos of C. capitata demonstrated the dynamic expression profile of these transcripts during early development [21]. Homologues of the principal sex-determination genes tra, tra-2 and $d s x$ have been sequenced in Bactrocera fruit flies $[17,22-24]$, as has $S x l$, which differs again from the Drosophila model by its maternal deposition in the egg. $S x l$ is also zygotically transcribed in the pre-blastoderm embryo in fruit flies of both genera Bactrocera and Ceratitis $[21,24]$.

For non-drosophilid insects, in contrast to the wellstudied model species Drosophila, different modes of regulation of the early stages of development and activation of the sex-determination pathway have been identified. In many dipterans, it appears that zygotic transcription from the Y-chromosome enables the female-specific sexdetermination gene transcripts, which are part of the maternal mRNA complement, to be replaced by malespecific transcripts, thus resetting cell memory [25]. This putative Y-chromosome transcript is the Dominant Male Determiner $(M)$, which has yet to be characterised, but the most likely target appears to be the TRA/TRA-2 based spliceosome, thereby effectively prohibiting the femalespecific splicing of both tra and $d s x$ pre-mRNA [24]. In Bombyx mori (Lepidoptera) with a ZZ:ZW sex-determination system where females are the heterogametic sex, a piRNA transcribed from the $\mathrm{W}$ chromosome has been identified as the dominant feminising factor [26].
Bactrocera tryoni or Queensland fruit fly is the major fruit fly pest species in Australia, exhibiting a wide host fruit range and geographic distribution; it is the primary target in Australia of pest management strategies, including sterile insect technique [SIT; [27]] and potentially incompatible insect technique [IIT; [28]]. Bactrocera jarvisi is a tephritid pest endemic to the north and east coast of Australia but has a narrower host range compared to that of B. tryoni. B. jarvisi is extremely useful in research because of its tractable laboratory maintenance, ability to form fertile hybrids with $B$. tryoni, and its possession of two Y-chromosome genetic markers, fundamental for discerning male and female embryos $[24,29]$. These features were exploited to perform qRT-PCR expression analysis of genes, previously identified as $D$. melanogaster sex-determination and cellularisation genes, using single, sexed embryos of both $B$. jarvisi and a B. tryoni line carrying the introgressed $B$. jarvisi $\mathrm{Y}$-chromosome [24], from $1 \mathrm{~h}$ to $9 \mathrm{~h}$ after egg laying (AEL). The demonstrated reciprocal compatibility of the male determiner in B. jarvisi and B. tryoni [29] anticipates strategies for optimising SIT and IIT in $B$. tryoni, such as the development of genetic sexing constructs [30] and embryonic lethality [31]. With established germ-line transformation protocols [32], these strategies may be transferable between species, supported by access to whole genome assemblies for both B. tryoni (GenBank accession No. JHQJ00000000) and $B$. jarvisi (Gilchrist et al., unpublished).

The embryonic progression of $B$. jarvisi from fertilisation to cellularisation takes seven hours. Expression analysis of $S x l$ and slam demonstrated that zygotic transcription has begun by $4 \mathrm{~h}$ post fertilisation, and affects sex-specific transcripts from $5 \mathrm{~h}$ in $B$. jarvisi and from about 8 hours in $B$. tryoni laboratory lines [24]. This tight time period of zygotic transcription and sex determination adds to the utility of $B$. jarvisi as a model for early embryonic studies. Therefore, we chose to analyse poly $\left(\mathrm{A}^{+}\right)$transcriptome data from sexed B. jarvisi embryos of 2-3h and 3-5h AEL to investigate three aspects of early development. One focused on the expression of sex-determination genes important to the Drosophila sex-determination pathway. The aim was to get a broader insight into the expression profiles of genes that have been co-opted to regulate the sex-determination pathway upstream of the primary genes conserved across tephritids and drosophilids. Secondly, we searched the $B$. jarvisi assembly for genes showing early zygotic expression in D. melanogaster, focusing on cellularisation genes, to identify homologues possessing early-acting promoters; and thirdly we used differential expression analysis to search for genes expressed differentially in male and female embryos, including candidate Y-chromosome transcripts, which are not maternally deposited and are only transcribed in males. 


\section{Methods}

\section{Fly Rearing}

B. jarvisi (BJ) laboratory stock was originally sourced from the Queensland Department of Agriculture, Fisheries and Forestry, Australia, and maintained at University of New South Wales and then University of Western Sydney at constant $25^{\circ} \mathrm{C}$, natural light and on artificial larval diet [33]. Flies were fed protein in the form of yeast hydrolysate and sugar one week after emergence, and two to four weeks later gravid females were induced to lay eggs into larval diet covered by perforated parafilm.

\section{Embryo collection and RNA and DNA extraction}

Embryos of two different age ranges were selected for sequencing: $2-3 \mathrm{~h}$ and 3-5h after egg laying (AEL). For each time period, it was necessary to separate male and female embryos based on a molecular marker on the Ychromosome, as there are no morphological characters to distinguish the sex of early embryos. Each embryo was individually processed for RNA extraction and DNA was isolated from these embryos for designation of sex. Embryos for RNA and DNA extraction were collected from $B$. jarvisi females by inducing egg laying through perforated parafilm coated with apple juice for a period of $1 \mathrm{~h}$ (first egg batch discarded), and then placed on moist fabric and maintained at $25^{\circ} \mathrm{C}$ and $70 \%$ humidity for the appropriate time. Individual embryos were placed in microcentrifuge tubes, $100 \mu \mathrm{L}$ TRIsure (Bioline Australia) added and embryos crushed using microtube pestles (SSI). Samples were incubated at room temperature (RT) for several minutes and then stored at $-80^{\circ} \mathrm{C}$.

Extraction of RNA from single embryos followed the TRIsure method, scaled down to $100 \mu \mathrm{L}$ TRIsure per embryo and further modified for use with Phase lock gel tubes (PLG; 5PRIME, Germany). Here, $40 \mu \mathrm{L}$ DEPC- $\mathrm{H}_{2} \mathrm{O}$ and $70 \mu \mathrm{L}$ chloroform (Sigma, St Louis, MO, USA) were added to the tissue in TRIsure before transferring to the PLG tubes and centrifuging for $5 \mathrm{~min}$ at $12000 \mathrm{~g}$ at RT, as per the PLG tube manufacturer's recommendations. The upper aqueous phase was pipetted into fresh tubes containing $5 \mu \mathrm{g}$ glycogen (Roche) and $70 \mu \mathrm{L}$ isopropanol (Sigma) and mixed. The samples were incubated at RT for $10 \mathrm{~min}$, then at $-20^{\circ} \mathrm{C}$ for several hours, before centrifugation at $12000 \mathrm{~g}$ at $4-8^{\circ} \mathrm{C}$ for $10 \mathrm{~min}$. The supernatant was decanted and the RNA was washed twice in $75 \%$ ethanol, air dried and resuspended in $15 \mu \mathrm{L}$ nucleasefree water. RNA integrity was examined by non-denaturing agarose gel electrophoresis of $1 \mu \mathrm{L}$ of each $15 \mu \mathrm{L}$ sample. Samples with poor yield or degraded RNA were discarded; acceptable samples were pooled (see below) and stored at $-80^{\circ} \mathrm{C}$ until required.

DNA is found in the interphase of the TRIsure / Chloroform mix after centrifugation, below the gel phase. With the aqueous RNA layer removed, $1 \mathrm{M}$ Tris at $\mathrm{pH}$ 7.6-8.0 was added to the organic phase, mixed and incubated at RT for more than $15 \mathrm{~min}$. Following centrifugation for $10 \mathrm{~min}$ at $12000 \mathrm{~g}$, the aqueous layer containing DNA was transferred to a new tube, ethanol precipitated and resuspended in $10 \mu \mathrm{L}$ nuclease-free water.

Multiplex PCR to designate sex was performed on the DNA from each embryo under conditions reported in Morrow et al. [24], using BjY2traB and BjY2traDrev primers to amplify the Y-chromosome fragment (227bp) and primers SxlRTFor 1 and SxlRTRev1 to amplify a 280bp product from both sexes (see Additional File 1). When multiplex PCR failed, single target PCR with primers BjY2traA and BjY2traDrev (311bp amplicon) and $S x l$ (as above) was carried out. Many samples failed to amplify with any set of primers probably due to the low copy number; these were discarded.

RNA preparations from the two time points, following sex designation, were pooled to create two male samples at 3-5h AEL (26 and 23 embryos), two female samples at 3-5h AEL (27 and 17 embryos), two male samples at 2-3h AEL (28 and 24 embryos) and two female samples at 2-3h AEL (12 and 24 embryos). Pooled samples were ethanol precipitated, washed six times in $75 \%$ ethanol and resuspended in $30 \mu \mathrm{L}$ DEPC- $\mathrm{H}_{2} \mathrm{O}$. Quality of the RNA preparations was ascertained by Nanodrop spectrophotometry, Qubit DNA and gel electrophoresis (Additional File 2). $\operatorname{Poly}\left(\mathrm{A}^{+}\right)$selection, reverse transcription and library construction using TruSeq RNA LT kit followed the Illumina RNA-Seq protocol and were performed at the Next Generation Sequencing Facility, Hawkesbury Institute for the Environment, UWS (Richmond, Australia). Eight paired-end libraries (300bp), comprising the two female samples and two male samples at both 2-3h AEL and 3-5h AEL time points, were sequenced on two lanes of Illumina HiSeq 1500. The sequencing output of $2 \times 100 \mathrm{bp}$ was demultiplexed and provided as two paired fastq files for each sample.

\section{Transcriptome assembly and annotation}

The raw sequence data were quality trimmed and filtered using CLC Genomics Workbench ver.6 (CLCbio), with parameters allowing 2 ambiguous nucleotides, minimum read length of 50 nucleotides and error probability limit of 0.05 applied to Phred quality scores according to the modified Mott trimming algorithm (see CLC Genomics Workbench v6 manual). De novo assembly of reads from all eight samples combined was performed with CLC Genomics using default parameters (herein referred to as "CLC assembly"). Within the CLC workbench, the entire CLC assembly was queried against the NCBI non-redundant (nr) protein database (downloaded October 2013) using blastx (cut-off E-value 1E-3). The subset of contigs with E-value cut-off of $1 \mathrm{E}-3$ were then used as a query in 
blastx searches of the NCBI nr protein database followed by gene ontology assignment, implemented in Blast2GO [34].

The CLC assembly was interrogated for twenty-eight genes involved in sex-determination processes in Drosophila spp. (GO:0007530) and four genes involved in cellularisation. First, the genes were sought amongst the top BLAST hits and accepted if the E-value was $<1 \mathrm{E}-3$. For those genes that did not find a match to B. jarvisi sequences in the CLC assembly, D. melanogaster and C. capitata sequences from GenBank were aligned in Mega 5.05 [35], and, where possible, a highly conserved section of the homologous $C$. capitata gene was used in a motif search of the CLC assembly contigs. The contigs with matches were then realigned with the D. melanogaster or C. capitata sequences in Mega 5.05 to confirm homology and to determine the length of the ORF. Consensus sequences for genes with multiple contigs were compiled for expression analysis.

\section{Differential expression}

RNA-Seq analysis was performed on the CLC assembly within CLC Genomics Workbench. The trimmed paired reads from each sample were mapped to the CLC assembly using default parameters which include minimum length (0.9) and similarity (0.8) fractions and expression values reported as RPKM (Reads Per Kilobase of exon model per Million mapped reads). Patterns of expression of known D. melanogaster sex-determination gene homologues ( $S x l$, tra, tra-2 and $d s x$ ) from qRT-PCR were compared to the RNA-Seq output. The cellularisation gene slam was shown to be transcribed between three to four hours (AEL) by qRT-PCR analysis [24] and the sisA transcript was identified in this study (see results). In D. melanogaster, slam is expressed in nuclear cycle 11 and sis $A$ is transcribed in nuclear cycle 8. The expression levels of these genes were assessed to determine if zygotic transcription was proceeding in the 2-3h embryos, and to determine an appropriate RPKM value threshold, over which expression values are likely to indicate maternally derived transcripts.

Differential expression comparisons were run on fourteen combinations of the eight samples across time and sex, and the RPKM values were quantile normalised and fold changes calculated. The proportions-based (Baggerley's) test was applied to normalised expression values, differences were selected when false discovery rate (FDR) -corrected $\mathrm{p}<0.001$. To investigate zygotic expression, differential expression output was filtered to select transcripts up-regulated in 3-5h versus 2 -3h embryos and male versus female embryos. Selections were filtered further by restricting the mean normalised RPKM values in the baseline sample to a variable number, based on assessment of slam and sisA transcript expression.

\section{Results}

A developmental time series of $B$. jarvisi transcript levels of homologues of the D. melanogaster genes, Sxl, tra, tra2, $d s x$ and slam, enabled us to choose two developmental time periods as targets for transcriptome analysis [24]. The period 2-3h AEL was selected because it fell in the phase as zygotic transcription was just beginning, while containing enough nuclei to confidently determine the sex of the embryo; and 3-5h AEL to cover the phase when zygotic transcription was proceeding, including the time when $M$ was expected to be active.

\section{Transcriptome assembly}

The Illumina RNA-Seq output comprised eight B. jarvisi samples with a range of 53 million to 112 million reads prior to quality trimming and filtering (Additional File 3). The filtered sequence reads from all eight samples were assembled de novo by CLC Genomics and produced 61,223 contigs of average size $699 \mathrm{bp}$ (Table 1 ). The majority of contigs were between 200 and 500bp (64.6\%), while $34.2 \%$ were over $500 \mathrm{bp}$ in length. Homology searches using blastx (NCBI nr protein database, October 2013) returned 23,518 sequences (E-value < 1E-3), including 260 (1.1\%) sequences matching Bactrocera species; 16,246 (69.1\%) matching sequences from Ceratitis spp., mostly from C. capitata $(16,209)$; and 2,596 (11.0\%) sequences matching Drosophila species. Of those sequences homologous to Bactrocera, the majority corresponded to $B$. dorsalis (156), B. oleae (64) and B. tryoni (29).

The CLC assembly dataset was too large to annotate using Blast2GO, therefore the subset of 23,518 contigs with Evalues lower than 1E-3, was uploaded into Blast2GO [34] for BLAST matching and gene ontology (GO). BLAST using the September 2013 NCBI invertebrate reference library matched 19,449 sequences (82.7\%); the top hits distributed primarily among Drosophila spp., most prominently D. virilis, D. mojavensis and D. melanogaster (Additional File 4).

Gene ontology (GO) analysis annotated 8,864 transcripts (45.6\%), categorised into 37 functional groups within the classes molecular function, cellular component and biological process (Figure 1). The largest representations were in "binding" and "catalytic activity" (molecular function), "cell" (cellular component) and "cellular process", "metabolic process" and "single-organism process" (biological process). Within biological processes, 1,108 sequences mapped to developmental processes (GO:0032502); 274 sequences were developmental processes involved in reproduction (GO:0003006) and 13 sequences mapped to sex determination (GO:0007530).

\section{RNA expression analysis and validation}

Within the RNA-Seq module of CLC Genomics, each trimmed library was mapped to the CLC assembly and 
Table 1 CLC de novo assembly of transcripts from all eight libraries combined.

\begin{tabular}{lllllll}
\hline Summary Statistics & Count & Average length & Total bases & Nucleotide distribution & Count & Frequency \\
\hline Reads & $604,738,912$ & 99.24 & $60,014,654,938$ & Adenine (A) & $13,031,025$ & $30.40 \%$ \\
Matched & $476,830,889$ & 99.24 & $47,320,571,906$ & Cytosine (C) & $7,923,401$ & $18.50 \%$ \\
Not matched & $127,908,023$ & 99.24 & $12,694,083,032$ & Guanine (G) & $7,925,335$ & $18.50 \%$ \\
Reads in pairs & $418,126,768$ & 154.99 & & Thymine (T) & $13,018,385$ & $30.40 \%$ \\
Broken paired reads & $58,704,121$ & 98.54 & & Any nucleotide (N) & 899,616 & $2.10 \%$ \\
\hline Contig measurements & Length & & & & \\
\hline Count & 61,223 & & & & \\
N75 & 434 & & & & \\
N50 & 1,138 & & & & \\
N25 & 2,697 & & & \\
Minimum contig (bp) & 114 & & & \\
Maximum contig (bp) & 17,400 & & & & \\
Average (bp) & 699 & & & & \\
Total (bp) & $42,797,762$ & & & & \\
\hline
\end{tabular}

levels of expression were recorded as normalised RPKM values. Validation of the assembly and mapping was performed. Principal components analysis of the eight samples showed samples clustering according to age, except for two samples, BJ1[male 3-5h] and BJ5[male 2-3h], which clustered together and not with their replicates
(Figure 2). To investigate this discrepancy, the comparative expression levels over the two time points for the genes Sxl, tra, tra-2, $d s x$ and slam [24] served as controls to validate the RPKM values. As there is no difference between male and female embryos in expression pattern for $S x l, t r a-2$ and female-specific tra and $d s x$ over the

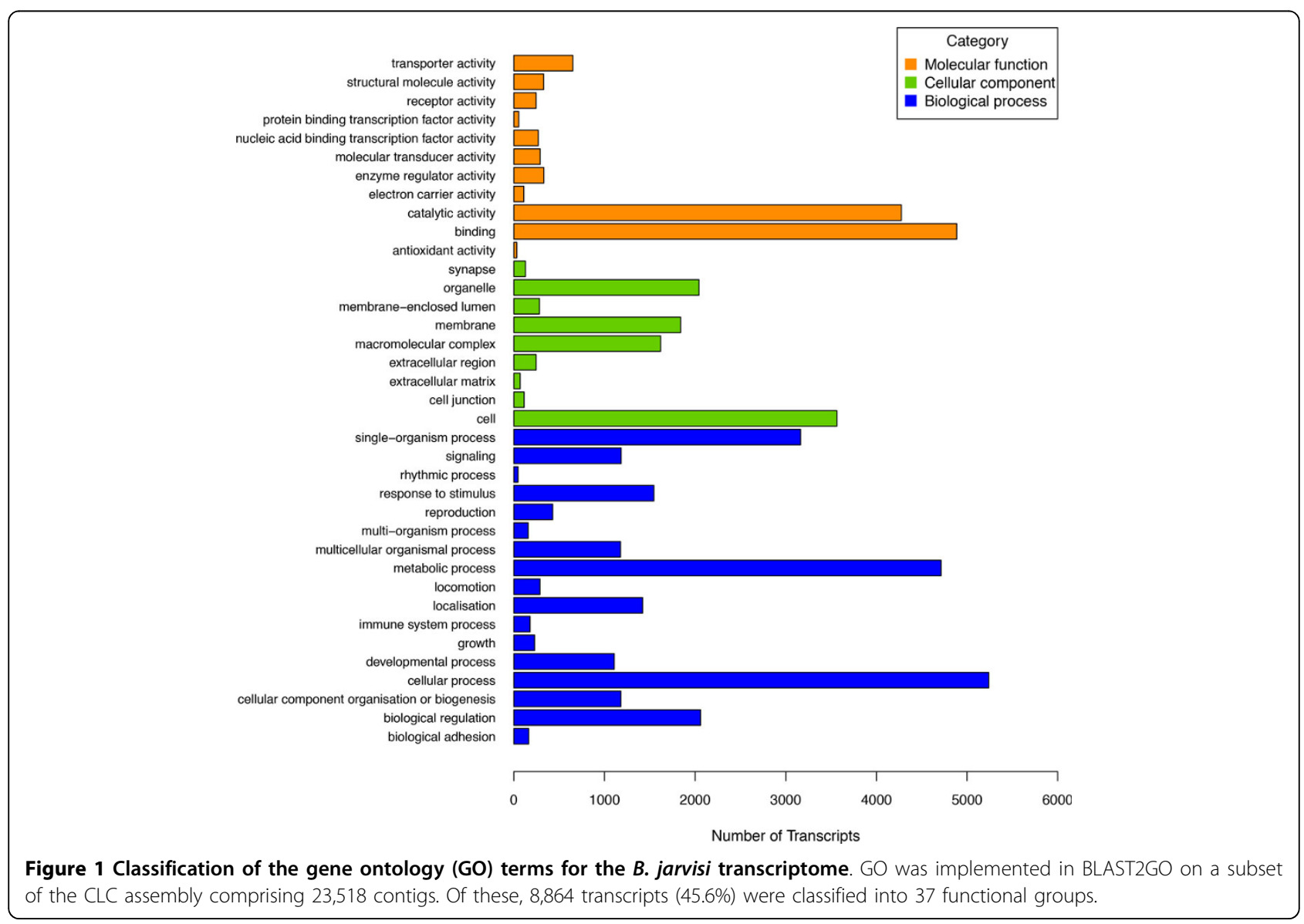




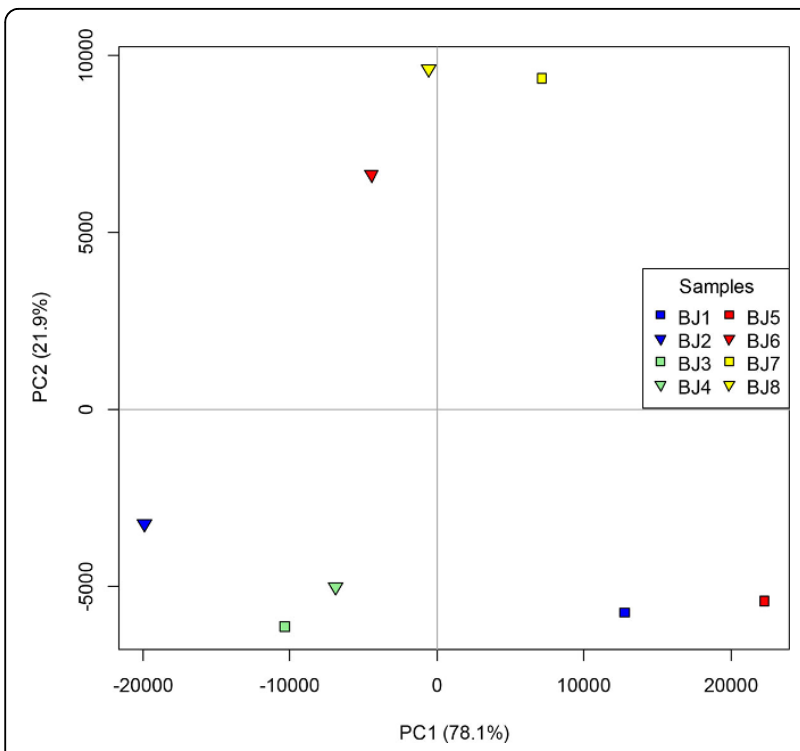

Figure 2 Principal co-ordinates analysis of the eight transcriptome samples mapped to the CLC assembly. Samples are male 3-5h (blue), female 3-5h (green), male 2-3h (red) and female 2-3h (yellow).

first 5h, differential expression across the two developmental periods, irrespective of sex, was examined. However, the results for $S x l$ (maternal and zygotic expression) and slam (zygotic only) were most useful because both exhibit an increase in transcript abundance over this time course [24]. Sxl RPKM values indicated that BJ1[male $3-5 \mathrm{~h}$ ] had low expression levels equivalent to the $2-3 \mathrm{~h}$ samples, rather than the higher levels of the other $3-5 \mathrm{~h}$ samples. The RPKM values for slam, a zygotically transcribed gene validated by RT-PCR, were low in BJ1[male 3-5h] (36 RPKM) compared to the other three 3-5h samples (299-461 RPKM), and were very low in three $2-3 \mathrm{~h}$ samples (0.25-2.02 RPKM) with the fourth 2-3h sample, BJ8[female 2-3h] showing a somewhat higher level (13 RPKM). The difference in RPKM values over time periods was not significant for slam, unless BJ1[male 3-5h] alone or $\mathrm{BJ} 1$ [male $3-5 \mathrm{~h}$ ] and $\mathrm{BJ} 5$ [male 2-3h] were excluded (FDR $<0.001)$. A similar phenomenon was also observed for sisA, run, gro, female lethal d $(f l(2) d)$, emc and $d p n$ (see sex-determination gene analysis, below), where each gene exhibited increased transcription in the other three 3-5h embryos (and significant up-regulation following exclusion of BJ1[male 3-5h] and BJ5[male 2-3h]; FDR $<0.001)$.

Taken together, these results suggest two features of the samples that may cause discrepancies (Figure 2): firstly, the age range of one hour in the 2-3h samples and 2 hours in 3-5h samples may have introduced variability, especially in the expression of early or rapidly increasing transcripts, such as slam, but may also have resulted in detection of early zygotic activity of $e m c, d p n$ and run (for example, if sample BJ8 included some more developed embryos). Secondly, the expression levels of $\mathrm{BJ} 1$ [male 3-5h] transcripts appeared to result from either a preponderance of embryos at the earlier end of the range, or from a sample in which many embryos did not survive to develop to the full 3-5h, thus producing a weaker increase in expression levels for those genes undergoing zygotic transcription, or a higher apparent expression value for maternal transcripts undergoing degradation in older embryos. Therefore, differential analysis was performed both with and without the BJ1[male 3-5h] and BJ5[male 2-3h] samples, employing 14 combinations to identify changes in transcript expression. The number of contigs differentially expressed $(\mathrm{FDR}<0.001)$, shows clearly that when time comparisons were undertaken, greater differences were recovered in the absence of samples $B J 1$ [male 3-5h] and $\mathrm{BJ} 5$ [male 2-3h], with $\mathrm{BJ} 1$ responsible for the greater impact. As a result, and to maintain consistency, the analyses of "Data mining for differentially expressed transcripts", below, were performed with and without $\mathrm{BJ} 1$ and BJ5.

\section{Sex-determination gene expression}

The CLC assembly was examined for the 28 Drosophila genes categorised as sex determination group (GO:0007530). Using the blastx annotation of the CLC assembly, the 13 contigs classified as sex-determination in the BLAST2GO pipeline and additional motif searches, the contigs were screened and 20 genes secured a contig match (Additional File 5A, Table 2A). The matching contigs were verified by alignment with the database nucleotide sequence and the ORF determined. This process also helped to assess the quality of assembly through identification of complete or partial open reading frames (ORFs). Only ten transcripts contained a complete ORF in a single contig and two more single contigs covered over $90 \%$ of the ORF (for $S x l$ and $f l(2) d)$. Eight genes were traversed by two to 11 contigs which covered more than $90 \%$ of the ORF (Additional File $5 \mathrm{~A}$ ), thus consensus sequences were produced by combining these contigs manually. The limitations of assembly in some cases appeared to be the result of different variants or alleles, but may be due to insufficiently stringent quality control.

Four of these genes - Sxl, tra-2, female-specific tra and $d s x$ - are recognised maternal transcripts in $B$. jarvisi that exhibited either no substantial change over the $2-5 \mathrm{~h}$ period or, for $S x l$, increased expression between 3 and 4 h [24]. RNA-seq of 2-3h and 3-5h embryos detected high levels of these transcripts, except for $d s x$ where low levels (normalised $\mathrm{RPKM}<1$ ) of not always complete transcripts were detected in all samples. No significant change in expression from one time sample to the next was recorded. Other 
Drosophila sex-determination genes detected by BLAST homology and expressed in 2-3h embryos are likely to represent maternally derived transcripts. This includes $d a$, $f l(2) d$, fruitless F (fru), gro, hopscotch (hop), Mes-4 (Mes-4), ovarian tumour (otu), sans fille (snf) and virilizer (vir) (Table 2A). Other transcripts that are first transcribed in the zygote include $d p n$ and $e m c$, which have low RPKM in 2-3h samples (0.25-1.06 and 0.44-2.5 respectively); for emc, the apparent early zygotic transcription contrasted with its maternal deposition in Drosophila.

\section{Cellularisation genes}

Some genes involved in cellularisation in Drosophila species are activated early in embryonic development.
No slam was detected in 0 -3h embryos by qRT-PCR [24], but transcript levels rapidly increased over the next three hours of development. Differential expression of slam transcripts revealed a mean RPKM of 4.1 in $2-3 \mathrm{~h}$ embryos and 305.3 in 3-5h embryos (Table 2B), signifying a substantial increase (76-fold) over the two time periods, but also showing that slam is zygotically expressed at low levels earlier than $3 \mathrm{~h}$. Three additional cellularisation genes sryo, nullo and bnk were sought through BLAST annotation and motif searches of the CLC assembly (Additional File $5 \mathrm{~B}$ ). A potential match was found for nullo with $24.6 \%$ identity across 213 amino acids with Drosophila simulans nullo (Acc. No. DSY44733) and 57\% identity over a conserved stretch of

Table 2 Expression profiles of the 20 sex-determination (A) and three cellularisation (B) transcripts identified in the $B$. jarvisi CLC assembly. Expression in each sample BJ1 - BJ8 is reported as the normalised RPKM value from the comparison of early samples (BJ5-8) to late samples (BJ1-4). Also refer to Additional File 5.

\begin{tabular}{|c|c|c|c|c|c|c|c|c|c|c|c|c|c|c|c|c|}
\hline \multirow[b]{3}{*}{$\begin{array}{l}\text { A. Sex } \\
\text { determination } \\
\text { genes }\end{array}$} & \multirow[b]{3}{*}{ contig no. } & \multirow[b]{3}{*}{$\begin{array}{l}\text { contig } \\
\text { size }\end{array}$} & \multirow[b]{3}{*}{$\begin{array}{l}\text { ORF } \\
\text { size }\end{array}$} & \multicolumn{4}{|c|}{ "early" samples } & \multicolumn{4}{|c|}{ "late" samples } & \multicolumn{2}{|c|}{$\begin{array}{l}\text { Significant up or } \\
\text { down-regulation } \\
\text { Comparison }\end{array}$} & \multicolumn{3}{|c|}{$\begin{array}{l}\text { Validated } \\
\text { expression } \\
\text { profile\# }\end{array}$} \\
\hline & & & & \multicolumn{2}{|c|}{ male } & \multicolumn{2}{|c|}{ female } & \multicolumn{2}{|c|}{ male } & \multicolumn{2}{|c|}{ female } & \multirow[b]{2}{*}{$\begin{array}{l}\text { early } \\
\text { vs } \\
\text { late }\end{array}$} & \multirow[b]{2}{*}{$\begin{array}{l}\text { early vs late } \\
\text { (exclude BJ1 } \\
\text { and BJ5) }\end{array}$} & \multirow[b]{2}{*}{$\mathrm{Bj}$} & \multirow[b]{2}{*}{ Cc } & \multirow[b]{2}{*}{$\mathrm{Dm}$} \\
\hline & & & & BJ5 & BJ6 & BJ7 & BJ8 & BJ1 & $\mathrm{BJ} 2$ & BJ3 & BJ4 & & & & & \\
\hline doublesex F & consensus & 1839 & $321 a a$ & 0.42 & 0.19 & 0.17 & 0.22 & 0.55 & 0.04 & 0.09 & 0.03 & ns & $\mathrm{ns}$ & $M$ & $M$ & Z \\
\hline daughterless & 16154 & 4053 & 710aа & 3.03 & 3.39 & 9.88 & 9.94 & 1.51 & 4.52 & 4.26 & 4.53 & ns & ns & & & $M$ \\
\hline deadpan & 20953 & 3365 & 574aa & 0.28 & 0.25 & 0.26 & 1.06 & 0.95 & 10.92 & 11.18 & 24.97 & ns & 0.02 & & Z & Z \\
\hline degringolade & 2208 & 1737 & 385aа & 111.44 & 116.21 & 107.56 & 104.73 & 149.23 & 100.76 & 93.48 & 100.61 & ns & ns & & & $M$ \\
\hline $\begin{array}{l}\text { extra } \\
\text { macrochaetae }\end{array}$ & 10611 & 1230 & 265aa & 0.97 & 0.44 & 0.56 & 2.5 & 6.82 & 121.25 & 123.56 & 211.04 & ns & $<0.001$ & & & $M$ \\
\hline female lethal $d$ & 2854 & 2694 & 466aa * & 18.97 & 13.9 & 12.22 & 11.07 & 17.85 & 40.43 & 43.89 & 37.66 & 0.03 & $<0.001$ & & $M$ & $M$ \\
\hline fruitless & 29049 & 3175 & 675aа & 0.78 & 0.57 & 0.74 & 0.43 & 0.62 & 0.19 & 0.38 & 0.08 & ns & ns & & & \\
\hline groucho & 3466 & 4604 & 726аa & 16.34 & 7.42 & 13.32 & 12.69 & 13.69 & 51.44 & 59.52 & 94.36 & ns & $<0.001$ & & $M$ & $M$ \\
\hline hopscotch & 753 & 4443 & 1174aа & 53.5 & 20.8 & 23.27 & 19.15 & 40.31 & 15 & 20.17 & 23.18 & ns & ns & & & \\
\hline Mes-4 & 5091 & 5397 & 1557aa & 57.2 & 17.96 & 22.76 & 16.62 & 34.22 & 11.54 & 18.9 & 17.46 & ns & ns & & & \\
\hline ovarian tumor & consensus & 5276 & 1221aa & 31.52 & 22.68 & 62.4 & 46.29 & 22.6 & 20.46 & 26.52 & 16.5 & ns & $\mathrm{ns}$ & & & \\
\hline ovo (variant1) & 21175 & 255 & $86 a а$ * & 3.93 & 2.97 & 8.77 & 14.62 & 1.44 & 0.09 & 0.43 & 0.23 & ns & ns & & & \\
\hline ovo (variant 6) & consensus & 3076 & 739aа & 12.66 & 11.2 & 58.05 & 51.86 & 5.85 & 0.83 & 0.61 & 0.86 & ns & ns & & & \\
\hline runt & consensus & 2399 & 485aa & 0.74 & 0.22 & 0.13 & 5.4 & 11.8 & 65.22 & 58.82 & 36.12 & 0.04 & $<0.001$ & & & Z \\
\hline sans fille & 7153 & 1421 & 150aа & 35.59 & 80.26 & 75.06 & 85.98 & 43.71 & 65.1 & 55.76 & 50.27 & ns & 0.008 & & $M$ & \\
\hline scute & consensus & 817 & $272 \mathrm{aa}$ * & 0.11 & 0.15 & 0.12 & 0.17 & 0.07 & 0.05 & 0.05 & 0.08 & ns & ns & & & Z \\
\hline Sex-lethal & 2265 & 1667 & 444aa * & 23.93 & 21.11 & 23.7 & 22.69 & 22.68 & 38.45 & 42.67 & 64.18 & ns & 0.04 & $M$ & $M$ & Z \\
\hline sisterless A & 2508 & 264 & 89aa * & 71.62 & 39.09 & 29.59 & 38.58 & 89.42 & 180.25 & 216.18 & 294.05 & 0.03 & $<0.001$ & & Z & Z \\
\hline transformer $F$ & consensus & 3793 & 422aa & 27.3 & 22.76 & 18.43 & 18.49 & 28.18 & 17.12 & 17.29 & 21.22 & ns & $\mathrm{ns}$ & $M$ & $M$ & $M$ \\
\hline transformer-2 & 138 & 3165 & 251 aа & 48.16 & 44.99 & 41.74 & 44.01 & 47.47 & 37.15 & 37.52 & 39.39 & ns & ns & $M$ & $M$ & $M$ \\
\hline virilizer & consensus & 5999 & 1878aа & 27.4 & 31.59 & 38.11 & 36.09 & 30.89 & 28.63 & 32.69 & 27.83 & ns & $\mathrm{ns}$ & & & Z \\
\hline \multicolumn{17}{|l|}{$\begin{array}{l}\text { B. } \\
\text { Cellularisation } \\
\text { genes }\end{array}$} \\
\hline nullo & consensus & 1332 & 211aа & 1.15 & 2.52 & 0.13 & 15.34 & 8.59 & 162.77 & 125.03 & 45.74 & ns & 0.04 & & & Z \\
\hline serendipity $\alpha$ & 9105 & 5007 & $615 a a *$ & 13.31 & 11.32 & 10.66 & 9.64 & 12.42 & 4.22 & 3.5 & 4.31 & ns & $\mathrm{ns}$ & & Z & Z \\
\hline $\begin{array}{l}\text { slow as } \\
\text { molasses }\end{array}$ & 3824 & 5366 & 1568aa & 2.02 & 0.76 & 0.25 & 13.35 & 35.87 & 460.96 & 425.02 & 299.29 & ns & $<0.001$ & Z & Z & Z \\
\hline
\end{tabular}

*Partial ORF

\#Experimentally validated in B. jarvisi (Bj), C. capitata (Cc) and D. melanogaster (Dm); maternal (M) or zygotic (Z) expression 
28 amino acids. Other than nullo homologues from ten Drosophila species, there were no records of nullo in the NCBI database. This sequence displayed a similar expression profile as slam, with an 18-fold increase over time (Table 2B). Neither sry $\alpha$ nor bnk were detected through the BLAST annotation, but a motif search of a $50 \mathrm{bp}$ fragment of the C. capitata sry $\alpha$ gene (Acc. No. FJ460703) identified a single contig with a partial ORF of 615 amino acids, exhibiting $61.6 \%$ and $33.5 \%$ identity with $C$. capitata and $D$. melanogaster sry $\alpha$ respectively. The expression profile did not emulate that of a zygotic transcript, as transcript levels diminished 2-fold as the embryos aged.

\section{Data mining for differentially expressed transcripts}

For male/female comparisons, differentially expressed transcripts that adhered to criteria of low or zero female expression with higher expression in males (Table 3 comparisons A-G) were only found in comparisons B and $D$, identifying five and four transcripts, respectively (Table 4). Annotation revealed contaminant fungal, bacterial and ribosomal RNA sequences in seven of them. One 220bp sequence fragment was expressed 23-fold higher in male embryos at 2-3h of age: this sequence matched the $B$. dorsalis even-skipped (eve) pair-rule gene. However there was little support for this transcript to be considered a good $\mathrm{Y}$-chromosome candidate, as examination of the raw reads did not reveal either a pseudogene copy or alternative structure of eve; and inclusion of male and female data from 3-5h, showed higher expression in females as time proceeds, but a reduction in the male samples over time. To broaden the search for transcripts up-regulated in males, further comparisons were made to distinguish these transcripts; the most useful comparisons were I, L and N (Table 3). The differential expression between the 2-3h embryos and the $3-5 \mathrm{~h}$ embryos of both sexes (I) revealed significant change of expression in 2,313 transcripts, of which 1,213 were up-regulated in older embryos (FDR-corrected $\mathrm{p}<0.001)$ indicating zygotic transcription. The comparison of BJ2[male 3-5h] and BJ6[male 2-3h] (L) was not replicated, but was used to find the overlap with the sequences from I ( $\cap \mathrm{L})$. A subset of sequences expressed at no more than 50 RPKM in the $2-3 \mathrm{~h}$ samples was used to eliminate transcripts that are $20 \%$ more abundant than $\operatorname{sis} A$, a conservative estimate to minimise the elimination of candidate transcripts that were highly expressed in the 2-3h embryos. As a result, 341 transcripts common to both sets $(1,051$ and 673 sequences respectively) were extracted. To reduce the inclusion of false positives, comparison $\mathrm{N}$, highlighting up-regulated sequences in older female embryos, was added to exclude those sequences also transcribed strongly in female embryos. The resulting 105 sequences had top BLAST hits to 53 C. capitata sequences, 9 Drosophila, four transposon-related sequences from other arthropods, and 39 undetermined sequences (E-value $>1 \mathrm{E}-3$ or no match retrieved; Additional File 6 Additional File 7).

Two Drosophila genes involved in the maternal to zygotic transition, zelda (also known as vielfaltig, $v f l$ ) and smaug, are both expressed maternally, with smaug undergoing rapid degradation during nuclear cycles 3-6 [36]. Homologues of zelda, as identified by blastx identity (contig 13,492; Additional file 7), displayed a significant 5.8-fold increase in expression over time. The smaug transcript was identified by homology to $C$. capitata (Accession No. XP_004526919), and displayed an 11-fold decrease in transcript levels over the two time periods, emulating the expression profile of smaug in D. melanogaster.

\section{Discussion}

By utilising next-generation sequencing technology, we sequenced the transcriptome of $B$. jarvisi embryos during two developmental periods prior to blastoderm formation. Notably, by exploiting a molecular marker on the Y-chromosome of B. jarvisi males, this early and dynamic developmental period was examined independently in male and female embryos to distinguish transcripts differentially regulated between the sexes and over time. The identification, nucleotide sequence and broad expression profile of genes expressed in the early stages of sex determination provided the primary focus of this study and was approached in two ways: through homology-based identification of validated sex-determination genes from Drosophila and homologues from other tephritids including C. capitata; and expressionbased studies highlighting differential regulation of the $\operatorname{poly}\left(\mathrm{A}^{+}\right)$transcriptome over time and between the sexes. Attention was also given to identification and expression profiles of transcripts involved in the maternal to zygotic transition and cellularisation. This collection of transcriptome data is a substantial addition to the knowledge base of this fruit fly species and other closely-related Bactrocera species and it will form a basis for future laboratory-based analyses of gene expression in the early embryo.

\section{Different roles for sex-determination genes}

Sex-specific differences in expression levels of the four XSEs found in Drosophila were not observed for these genes in B. jarvisi. The four genes, sisA, sc, os and run are transcribed from both $\mathrm{X}$ chromosomes in Drosophila females, to communicate the female signal to $S x l$. Bearing in mind the non-sex-specificity of $S x l$ in tephritids, we identified B. jarvisi homologues of sisA, $s c$ and run, but not os, expressed in the early developmental stages; sis A and run increased 6- and 28-fold respectively over the two periods but did not differ in male 
Table 3 Summary of differential expression comparisons performed in CLC Genomics Workbench. Expression values were normalised and significant difference ascertained by Baggerley's test, with FDR-corrected $p$ value cutoff at $0.1 \%$. Contigs were selected for up-regulation in males over females, and 3-5h embryos over 2-3h embryos using normalised fold-change.

\begin{tabular}{|c|c|c|c|c|c|c|}
\hline & Comparison & Samples $^{1}$ & $\begin{array}{c}\text { No. of differentially } \\
\text { expressed contigs (FDR } \mathrm{p}< \\
0.001 \text { ) }\end{array}$ & $\begin{array}{l}\text { No. up-regulated in males } \\
\text { and/or older embryos }\end{array}$ & Additional filters & $\begin{array}{c}\text { No. of } \\
\text { transcripts of } \\
\text { interest }\end{array}$ \\
\hline & $\begin{array}{l}\text { Male/Female } \\
\text { comparisons }\end{array}$ & & & & & \\
\hline A & Late only (3-5h) & 1,2 vs 3,4 & 2 & 0 & & 0 \\
\hline B & $\begin{array}{l}\text { Late only (3-5h), excl. } \\
\text { BJ1 }\end{array}$ & 2 vs 3,4 & 21 & 9 & $\begin{array}{l}\text { tmean normalised RPKM } \\
\text { of female samples }<5\end{array}$ & 5 \\
\hline$C$ & Early only (2-3h) & 5,6 vs 7,8 & 73 & 4 & $\begin{array}{l}\text { tmean normalised RPKM } \\
\text { of female samples }<5\end{array}$ & 0 \\
\hline $\mathrm{D}$ & $\begin{array}{l}\text { Early only (2-3h), excl. } \\
\text { BJ5 }\end{array}$ & 6 vs 7,8 & 142 & 46 & $\begin{array}{l}\text { tmean normalised RPKM } \\
\text { of female samples }<5\end{array}$ & 4 \\
\hline $\mathrm{E}$ & Both (3-5h and 2-3h) & $\begin{array}{l}1,2,5,6 \text { vs } \\
3,4,7,8\end{array}$ & 0 & 0 & & \\
\hline $\mathrm{F}$ & $\begin{array}{l}\text { Both (3-5h and 2-3h) } \\
\text { excl. BJ1 and BJ5 }\end{array}$ & $\begin{array}{l}2,6 \text { vs } \\
3,4,7,8\end{array}$ & 0 & 0 & & \\
\hline \multirow[t]{2}{*}{ G } & $\begin{array}{l}\text { Both (3-5h and 2-3h) } \\
\text { excl. BJ1 }\end{array}$ & $\begin{array}{c}2,5,6 \text { vs } \\
3,4,7,8\end{array}$ & 0 & 0 & & \\
\hline & \multicolumn{6}{|l|}{ Time comparisons } \\
\hline $\mathrm{H}$ & $\begin{array}{l}\text { Both (Male and } \\
\text { female) }\end{array}$ & $\begin{array}{c}1,2,3,4 \text { vs } \\
5,6,7,8\end{array}$ & 283 & 86 & & \\
\hline । & $\begin{array}{c}\text { Both (Male and } \\
\text { female) excl. BJ1 and } \\
\text { BJ5 }\end{array}$ & $\begin{array}{c}2,3,4 \text { vs } \\
6,7,8\end{array}$ & 2313 & 1213 & $\begin{array}{l}\text { \#mean normalised RPKM } \\
\text { of } 2 \text {-3h samples }<50\end{array}$ & $1051^{*}$ \\
\hline J & $\begin{array}{l}\text { Both (Male and } \\
\text { female) excl. BJ1 }\end{array}$ & $\begin{array}{c}2,3,4 \text { vs } \\
5,6,7,8\end{array}$ & 1846 & 1128 & $\begin{array}{l}\text { \#mean normalised RPKM } \\
\text { of } 2-3 \mathrm{~h} \text { samples }<50\end{array}$ & 1019 \\
\hline K & Male only & 1,2 vs 5,6 & 14 & 2 & & \\
\hline $\mathrm{L}$ & $\begin{array}{l}\text { Male only excl. BJ1 } \\
\text { and BJ5 }\end{array}$ & 2 vs 6 & 1321 & 827 & $\begin{array}{l}\text { \#mean normalised RPKM } \\
\text { of } 2-3 \mathrm{~h} \text { samples }<50\end{array}$ & $673^{*}$ \\
\hline M & $\begin{array}{c}\text { Male only excl. BJ2 } \\
\text { and BJ6 }\end{array}$ & 1 vs 5 & 183 & 66 & & \\
\hline \multirow[t]{4}{*}{$\mathrm{N}$} & Female only & 3,4 vs 7,8 & 1980 & $862^{*}$ & & \\
\hline & Intersections & & & & & \\
\hline & $I \cap L$ & & & & & 341 \\
\hline & $(\mid \cap L)-(\mid \cap L \cap N)$ & & & & & 105 \\
\hline
\end{tabular}

${ }^{1}$ Samples BJ1- BJ8 are abbreviated to 1-8

†Allowed extra expression $(\mathrm{RPKM}<5)$ in female samples to cover any accidental inclusion of male embryos

\# Determined cutoff to be $20 \%$ higher than the mean expression, in 2-3h embryos, of sis $A$, a transcript expressed early in D. melanogaster

*Subsets of $\mathrm{I}, \mathrm{L}$ and $\mathrm{N}$ used in intersections

and female embryos, and $s c$ did not change over time. The $\mathrm{X}$ and $\mathrm{Y}$ chromosomes of B. tryoni are highly heterochromatic and the genes located on the Drosophila X chromosome are autosomal in tephritids [37], with no evidence that these genes play a role in sex determination. Of the four Drosophila XSEs, os is expressed latest (cycle 13) which may account for its absence in B. jarvisi embryos, or more likely, the $B$. jarvisi homologue was not sufficiently similar to the known Drosophila os sequences. However, while sis $A$ is expressed during cycle 8 and $s c$ during cycle 9 of Drosophila embryos [7], in $B$. jarvisi, sisA was highly expressed even in the younger embryos and may be displaying a rapid increase as development proceeds, and $s c$ was expressed very low in both time periods. Transcripts of sisA were not detected in C. capitata unfertilised eggs [21]. If $B$. jarvisi also conforms to this expression profile, then zygotic transcription of sisA is very early, well underway between 2 and $3 \mathrm{~h} \mathrm{AEL}$. An autosomal sex-determination gene in Drosophila expressed in the zygote is $d p n$, which complied with this pattern in $B$. jarvisi, and exhibited more than 30-fold increase in transcript levels from 2-3h to 35 h AEL.

Maternal transcripts in Drosophila include her, da, gro and $e m c$. Homologous sequence for her was not detected in B. jarvisi embryos, but $d a$ and gro were detected in 2$3 \mathrm{~h}$ embryos and gro exhibited a significant 6 -fold increase over time. In C. capitata, gro transcripts were found to be 
Table 4 Differential expression results showing significantly up-regulated transcripts in males over females in 3-5h samples (Comparison B) and 2-3h samples (Comparison D).

\begin{tabular}{|c|c|c|c|c|c|c|c|c|}
\hline \multirow{2}{*}{\multicolumn{2}{|c|}{$\begin{array}{l}\text { Comparison } \\
\text { B } \\
\text { Late only (3-5h), } \\
\text { excl. BJ1 }\end{array}$}} & \multicolumn{6}{|c|}{$\begin{array}{l}\text { Normalized expression values } \\
\text { (RPKM) }\end{array}$} & \multirow[t]{2}{*}{ Top BLAST hit } \\
\hline & & & male & fem & nale & & & \\
\hline Feature ID & $\begin{array}{l}\text { Gene } \\
\text { length }\end{array}$ & $\begin{array}{l}\text { Fold Change } \\
\text { (normalized } \\
\text { values) }\end{array}$ & $\mathrm{BJ} 2$ & BJ3 & BJ4 & $\begin{array}{c}\text { NCBI } \\
\text { Accession No. }\end{array}$ & E-value & Gene / Source \\
\hline contig 22537 & 1546 & -109.63 & 185.79 & 0.1 & 3.29 & EQB46020 & $7.85 E-55$ & $\begin{array}{l}\text { hypothetical protein CGLO_15009 [Colletotrichum } \\
\text { gloeosporioides Cg-14] (fungal pathogen of plants) }\end{array}$ \\
\hline contig 26828 & 407 & -90.35 & 145.56 & 0 & 3.22 & ELT94824 & $2.06 \mathrm{E}-40$ & $\begin{array}{c}\text { hypothetical protein CAPTEDRAFT_122939, partial } \\
\text { [Capitella teleta] (Annelid worm) }\end{array}$ \\
\hline contig 30721 & 521 & -44.23 & 68.22 & 0.39 & 2.7 & EEH16716 & $2.00 \mathrm{E}-28$ & $\begin{array}{c}\text { senescence-associated protein [Paracoccidioides } \\
\text { brasiliensis Pb03] > gi|225678434|gb|EEH16718.1| } \\
\text { (fungus) }\end{array}$ \\
\hline contig 47470 & 424 & -100.05 & 111.08 & 0.12 & 2.1 & XP_662849 & $3.93 \mathrm{E}-26$ & $\begin{array}{l}\text { hypothetical protein AN5245.2 [Aspergillus nidulans } \\
\text { FGSC A4] >gi|40742997|gb|EAA62187.1| (fungus) }\end{array}$ \\
\hline contig 49000 & 222 & -39.38 & 131.19 & 0.96 & 5.71 & EHK21457 & $3.99 \mathrm{E}-41$ & $\begin{array}{c}\text { hypothetical protein TRIVIDRAFT_53758, partial } \\
\text { [Trichoderma virens GV29-8] (fungus) }\end{array}$ \\
\hline \multicolumn{9}{|l|}{$\begin{array}{l}\text { Comparison } \\
\text { D }\end{array}$} \\
\hline \multicolumn{9}{|c|}{$\begin{array}{l}\text { Early only (2-3h), } \\
\text { excl. BJ5 }\end{array}$} \\
\hline & & & BJ6 & BJ7 & BJ8 & & & \\
\hline contig 31948 & 667 & -387.12 & 64.8 & 0.1 & 0.23 & CAJ30045 & $7.17 \mathrm{E}-28$ & $\begin{array}{l}\text { conserved hypothetical protein [Magnetospirillum } \\
\text { gryphiswaldense MSR-1] (bacterium) }\end{array}$ \\
\hline contig 3465 & 234 & -20.53 & 29.21 & 0.57 & 2.28 & XP_004522331 & $3.36 \mathrm{E}-45$ & $\begin{array}{l}\text { PREDICTED: } 60 \text { S acidic ribosomal protein P0-like } \\
\text { [Ceratitis capitata] >gi|20139848|sp|Q9U3U0.1| }\end{array}$ \\
\hline contig 41160 & 210 & -70.11 & 37.85 & 0.33 & 0.75 & WP_006574328 & $1.06 \mathrm{E}-16$ & $\begin{array}{c}\text { hypothetical protein [Pseudoflavonifractor capillosus] } \\
>\text { gi|150270408|gb|EDM97731.1 (bacterium) }\end{array}$ \\
\hline contig 567 & 220 & -23.73 & 33.44 & 2.82 & 0 & ACN91520 & $2.50 \mathrm{E}-16$ & eve [Bactrocera dorsalis] \\
\hline
\end{tabular}

maternal in origin [21]. Although confirmation is needed for the maternal input of $d a$ and gro in B. jarvisi, their presence in the 2-3hr transcriptomes would suggest that they are maternal transcripts. The expression profile of emc differed markedly from that expected for a maternal transcript and demonstrated in D. melanogaster; it displayed a significant 130-fold increase in transcript levels in 3-5h embryos, warranting further investigation of the potential functional role/s of this transcript in the developing embryo of Bactrocera relative to Drosophila.

Early zygotic transcripts as a source of promoters and enhancers for transgenic pest control strategies

A partial sequence for a putative homologue of the D. melanogaster gene nullo was identified by BLAST annotation, coupled with an expression profile emulating a known cellularisation gene, slam, which is also expressed from nuclear cycle 11 in $D$. melanogaster $[8,11]$. The sry $\alpha$ transcript, however, undetected in C. capitata prior to cellularisation [38] but reported as a maternal contribution by Gabrieli et al. [21], was found in B. jarvisi 2-3h embryos but had diminished levels in 3-5h embryos. Both slam and nullo, in addition to sis $A$, which also appeared to be expressed early in B. jarvisi, provide candidate promoter regions for use in transgenic constructs in the Bactrocera. The promoter/ enhancer regions of the sry $\alpha$ and slam genes have been used to create embryonic lethality systems in $C$. capitata [38] and the Anastrepha suspensa sry $\alpha$ homologue has been successfully applied to the construction of embryonic lethal [31] and female-specific lethal transgenes [30].

\section{Up-regulated transcripts in male embryos}

We were unable to confidently discern poly $\left(\mathrm{A}^{+}\right)$RNA that was more abundant in males and very low in females, and therefore potentially transcribed from the $\mathrm{Y}$ chromosome. The Y chromosome is highly heterochromatic [37], therefore few genes may be transcribed and these transcripts may not be collected in the poly $\left(\mathrm{A}^{+}\right)$ fraction of the transcriptome. However, the sequencing of the poly $\left(\mathrm{A}^{+}\right)$fraction of mRNA was an essential step for de novo assembly of male and female transcriptomes in the early Bactrocera embryo, providing numerous novel coding sequences with homology to known C. capitata and Drosophila spp. genes. These reads will add valuable sequence for analysis of the $B$. jarvisi draft 
genome assembly (Gilchrist et al. unpublished). Y-specific transcripts may yet be found by obtaining sufficient coverage to identify the Y-chromosome sequences by subtraction of the genome of female $B$. jarvisi from the male genome. Such an endeavour is underway, thus transcripts from the male samples could be mapped onto the Y chromosome and expression analysis repeated to determine up-regulated Y-specific transcripts as the embryos age.

\section{Conclusion}

To extend the results presented here, the next step will be to utilise whole genome sequence for B. jarvisi. Genome-guided transcriptome assembly was not feasible in this time frame, however applying this method may uncover transcripts that are differentially expressed or alternatively spliced or located on the $\mathrm{Y}$-chromosome. The differential analysis of the poly $\left(\mathrm{A}^{+}\right)$fraction has demonstrated the value of $B$. jarvisi as a study system for early embryonic development and sex determination. However, it has provided no evidence that $M$, the Dominant Male Determiner, might be a protein coding gene on the $\mathrm{Y}$ chromosome, and leaves open the possibility that any $\mathrm{Y}$-chromosome transcripts initiating male development may be non-protein coding. To identify potential candidates for $M$, it will be necessary to utilise the $B$. jarvisi embryo-sexing protocol and tight developmental time frame to also sequence the non-coding fractions of the transcriptome, that includes many regulatory RNAs [39]. The related pest fruit fly, B. tryoni, will soon have an annotated genome available, and so homology to the genes discovered here will allow valuable comparisons between the two species, which are reproductively compatible, such that the Y chromosome can be transferred between species in both directions. This work will be immediately useful for the identification and laboratory testing of cellularisation gene promoters, such as slam and nullo, for transgenic-based improvements to pest management strategies such as sterile insect technique (SIT) for both B. jarvisi and B. tryoni.

\section{Availability of supporting data}

Raw sequence reads have been submitted to the NCBI SRA (sequence read archive) database under accession number SRP046518.

\section{Additional material}

Additional File 1: PCR conditions and primer sequences used in this research.

Additional File 2: Quality and concentration of total RNA samples for transcriptome sequencing.

Additional File 3: Results of trimming and filtering poor quality reads in CLC Genomics Workbench.
Additional File 4: Species distribution of BLAST2GO top matches against a subset of the CLC assembly. A subset of 23,518 contigs were selected by e-value > 1E-3 match to NCBI nr database. Over $77 \%$ of the sequences matched Drosophila species.

Additional File 5: Sex-determination and cellularisation genes. A) The 28 Drosophila sex determination genes and the top BLAST hits against the nr database. Only 20 of these genes found a match in the CLC assembly. (B) Four cellularisation genes were sought, only nullo was found by the top blast hits. Other transcripts were identified by motif searches using $D$. melanogaster or $C$. capitata sequence as the query.

Additional File 6: A list of the 66 contigs up-regulated in older male embryos, after exclusion of contigs also up-regulated in older females. Normalised RPKM values are from comparison I (Table 3) with top BLAST hits to arthropod sequences from the NCBI nr database.

Additional File 7: A list of the Bactrocera jarvisi contigs upregulated in older embryos with BLAST matches to C. capitata and Drosophila genes (62 contigs). These were cross-referenced to FlyBase. The molecular and biological function of these genes and the expression profile in D. melanogaster are listed. Many genes have transcription or translation regulatory functions, but are transcribed in both males and females (see Additional File 6).

Competing interests

The authors declare that they have no competing interests.

\section{Authors' contributions}

The project was conceived and designed by D.C.A.S., M.F., M.R. and J.L.M.; J.L. M. performed the research, J.L.M. and A.S.G analysed the data; M.R. supplied all materials for sample preparation; J.L.M., M.R., D.C.A.S and M.F. contributed to writing the paper.

\section{Acknowledgements}

The authors are grateful to Paul Rymer and John Sved for assistance and guidance with analysis, Goran Lopaticki for maintaining the fly cultures, and Caroline Janitz from the HIE NGS Sequencing Facility for sample preparation advice and Illumina HiSeq sequencing. J.L.M. was supported by an Australian Postgraduate Award scholarship and F. G. Swain award, M.R. by research funds from the Hawkesbury Institute for the Environment, A.S.G by research funds from the University of New South Wales, and D.C.A.S and M.F by an Australian Research Council grant.

This article has been published as part of BMC Genetics Volume 15

Supplement 2, 2014: Development and evaluation of improved strains of insect pests for SIT. The full contents of the supplement are available online at http://www.biomedcentral.com/bmcgenet/supplements/15/S2.

Publication of this supplement was funded by the International Atomic Energy Agency. The peer review process for articles published in this supplement was overseen by the Supplement Editors in accordance with BioMed Central's peer review guidelines for supplements. The Supplement Editors declare that they have no competing interests.

\section{Authors' details}

${ }^{1}$ Hawkesbury Institute for the Environment, University of Western Sydney, Locked Bag 1797, Penrith, NSW 2751, Australia. 'Evolution and Ecology Research Centre, School of Biological, Earth and Environmental Sciences, University of New South Wales, Sydney, NSW 2052, Australia.

Published: 1 December 2014

\section{References}

1. Tadros W, Lipshitz HD: The maternal-to-zygotic transition: a play in two acts. Development 2009, 136(18):3033-3042.

2. Tadros W, Goldman AL, Babak T, Menzies F, Vardy L, Orr-Weaver T, Hughes TR, Westwood JT, Smibert CA, Lipshitz HD: SMAUG Is a Major Regulator of Maternal mRNA Destabilization in Drosophila and Its Translation Is Activated by the PAN GU Kinase. Developmental Cell 2007, 12(1):143-155. 
3. Bushati N, Stark A, Brennecke J, Cohen SM: Temporal Reciprocity of miRNAs and Their Targets during the Maternal-to-Zygotic Transition in Drosophila. Curr Biol 2008, 18(7):501-506.

4. Arbeitman MN, Furlong EEM, Imam F, Johnson E, Null BH, Baker BS, Krasnow MA, Scott MP, Davis RW, White KP: Gene expression during the life cycle of Drosophila melanogaster. Science 2002, 297(5590):2270-2275.

5. ten Bosch JR, Benavides JA, Cline TW: The TAGteam DNA motif controls the timing of Drosophila pre-blastoderm transcription. Development 2006, 133(10):1967-1977.

6. Liang $\mathrm{H}-\mathrm{L}$, Nien C-Y, Liu H-Y, Metzstein MM, Kirov N, Rushlow C: The zincfinger protein Zelda is a key activator of the early zygotic genome in Drosophila. Nature 2008, 456(7220):400-403.

7. Erickson JW, Cline TW: A bZIP protein, sisterless-a, collaborates with bHLH transcription factors early in Drosophila development to determine sex. Genes Dev 1993, 7(9):1688-1702.

8. Lecuit T, Samanta R, Wieschaus E: slam Encodes a Developmental Regulator of Polarized Membrane Growth during Cleavage of the Drosophila Embryo. Developmental Cell 2002, 2(4):425-436.

9. Stein JA, Broihier HT, Moore LA, Lehmann R: Slow as Molasses is required for polarized membrane growth and germ cell migration in Drosophila. Development 2002, 129(16):3925-3934.

10. Schejter $E D$, Wieschaus $\mathrm{E}$ : bottleneck acts as a regulator of the microfilament network governing cellularization of the Drosophila embryo. Cell 1993, 75(2):373-385.

11. Rose LS, Wieschaus E: The Drosophila cellularization gene nullo produces a blastoderm-specific transcript whose levels respond to the nucleocytoplasmic ratio. Genes Dev 1992, 6:1255-1268.

12. Ruiz MF, Eirin-Lopez JM, Stefani RN, Perondini AL, Selivon D, Sanchez L: The gene doublesex of Anastrepha fruit flies (Diptera, Tephritidae) and its evolution in insects. Dev Genes Evol 2007, 217(10):725-731

13. Ruiz MF, Milano A, Salvemini M, Eirin-Lopez JM, Perondini ALP, Selivon D, Polito C, Saccone G, Sanchez L: The Gene Transformer of Anastrepha Fruit Flies (Diptera, Tephritidae) and Its Evolution in Insects. PLoS One 2007 2(11):e1239

14. Sarno F, Ruiz MF, Eirín-Lápez JM, Perondini AL, Selivon D, Sànchez L: The gene transformer-2 of Anastrepha fruit flies (Diptera, Tephritidae) and its evolution in insects. BMC Evol Biol 2010, 10(1):140.

15. Salz HK, Erickson JW: Sex determination in Drosophila: The view from the top. Fly 2010, 4(1):60-70.

16. Penalva LO, Sanchez L: RNA binding protein sex-lethal $(S x l)$ and control of Drosophila sex determination and dosage compensation. Microbiol Mol Biol Rev 2003, 67(3):343-359.

17. Lagos D, Ruiz MF, Sanchez L, Komitopoulou K: Isolation and characterization of the Bactrocera oleae genes orthologous to the sex determining Sex-lethal and doublesex genes of Drosophila melanogaster. Gene 2005, 348:111-121.

18. Saccone G, Peluso I, Artiaco D, Giordano E, Bopp D, Polito LC: The Ceratitis capitata homologue of the Drosophila sex-determining gene Sex-lethal is structurally conserved, but not sex-specifically regulated. Development 1998, 125(8):1495-1500.

19. Meise M, Hilfiker-Kleiner D, Dubendorfer A, Brunner C, Nothiger R, Bopp D: Sex-lethal, the master sex-determining gene in Drosophila, is not sexspecifically regulated in Musca domestica. Development 1998, 125(8):1487-1494.

20. Gomulski LM, Dimopoulos G, Xi ZY, Soares MB, Bonaldo MF, Malacrida AR, Gasperi G: Gene discovery in an invasive tephritid model pest species, the Mediterranean fruit fly, Ceratitis capitata. BMC Genomics 2008, 9:243.

21. Gabrieli P, Falaguerra A, Siciliano P, Gomulski LM, Scolari F, Zacharopoulou A, Franz G, Malacrida AR, Gasperi G: Sex and the single embryo: early development in the Mediterranean fruit fly, Ceratitis capitata. BMC Dev Biol 2010, 10:12.

22. Lagos D, Koukidou M, Savakis C, Komitopoulou K: The transformer gene in Bactrocera oleae: the genetic switch that determines its sex fate. Insect Mol Biol 2007, 16(2):221-230.

23. Shearman DCA, Frommer M: The Bactrocera tryoni homologue of the Drosophila melanogaster sex-determination gene doublesex. Insect $\mathrm{Mol}$ Biol 1998, 7(4):355-366.

24. Morrow $J$, Riegler M, Frommer M, Shearman DCA: Expression patterns of sex-determination genes in single male and female embryos of two Bactrocera fruit fly species during early development. Insect Mol Biol 2014 , in press, doi: 10.1111/imb.12123.
25. Pane A, Salvemini M, Delli Bovi P, Polito C, Saccone G: The transformer gene in Ceratitis capitata provides a genetic basis for selecting and remembering the sexual fate. Development 2002, 129(15):3715-3725.

26. Kiuchi T, Koga H, Kawamoto M, Shoji K, Sakai H, Arai Y, Ishihara G, Kawaoka S, Sugano S, Shimada T: A single female-specific piRNA is the primary determiner of sex in the silkworm. Nature 2014, 509(7502):633-636.

27. Andrewartha HG, Monro J, Richardson NL: The use of sterile males to control populations of Queensland fruit fly Dacus tryoni (Frogg.)(Diptera: Tephritidae) II. Field experiments in New South Wales. Aust J Zool 1967, 15(3):475-499.

28. Boller EF, Russ K, Vallo V, Bush GL: Incompatible races of European cherry fruit fly, Rhagoletis cerasi (Diptera: Tephritidae), their origin and potential use in biological control. Entomol Exp Appl 1976, 20(3):237-247.

29. Shearman DCA, Frommer M, Morrow JL, Raphael KA, Gilchrist AS: Interspecific Hybridization as a Source of Novel Genetic Markers for the Sterile Insect Technique in Bactrocera tryoni (Diptera: Tephritidae). J Econ Entomol 2010, 103(4):1071-1079.

30. Schetelig MF, Handler AM: A transgenic embryonic sexing system for Anastrepha suspensa (Diptera: Tephritidae). Insect Biochem Mol Biol 2012, 42(10):790-795.

31. Schetelig MF, Handler AM: Strategy for enhanced transgenic strain development for embryonic conditional lethality in Anastrepha suspensa. Proc Natl Acad Sci USA 2012, 109(24):9348-9353.

32. Raphael KA, Shearman DCA, Streamer K, Morrow JL, Handler AM, Frommer M: Germ-line transformation of the Queensland fruit fly, Bactrocera tryoni, using a piggyBac vector in the presence of endogenous piggyBac elements. Genetica 2011, 139(1):91-97.

33. Meats A, Holmes HM, Kelly GL: Laboratory adaptation of Bactrocera tryoni (Diptera: Tephritidae) decreases mating age and increases protein consumption and number of eggs produced per milligram of protein. Bull Entomol Res 2004, 94(6):517-524.

34. Conesa A, Götz S, García-Gómez JM, Terol J, Talón M, Robles M: Blast2GO: a universal tool for annotation, visualization and analysis in functional genomics research. Bioinformatics 2005, 21(18):3674-3676.

35. Tamura K, Peterson D, Peterson N, Stecher G, Nei M, Kumar S: MEGA5 molecular evolutionary genetics analysis using maximum likelihood, evolutionary distance, and maximum parsimony methods. Mol Biol Evol 2011, 28(10):2731-2739.

36. Fisher B, Weiszmann R, Frise E, Hammonds A, Tomancak P, Beaton A, Berman B, Quan E, Shu S, Lewis S, et al: BDGP insitu homepage 2012 [http:// insitu.fruitfly.org/cgi-bin/ex/insitu.pl].

37. Zhao JT, Frommer M, Sved JA, Zacharopoulou A: Mitotic and polytene chromosome analyses in the Queensland fruit fly, Bactrocera tryoni (Diptera: Tephritidae). Genome 1998, 41(4):510-526.

38. Schetelig MF, Caceres C, Zacharopoulou A, Franz G, Wimmer EA: Conditional embryonic lethality to improve the sterile insect technique in Ceratitis capitata (Diptera: Tephritidae). BMC Biology 2009, 7(1):4.

39. Mattick JS, Makunin IV: Non-coding RNA. Human molecular genetics 2006, 15(suppl 1):R17-R29.

\section{doi:10.1186/1471-2156-15-S2-S7}

Cite this article as: Morrow et al:: Comprehensive transcriptome analysis of early male and female Bactrocera jarvisi embryos. BMC Genetics 2014 15(Suppl 2):S7.

\section{Submit your next manuscript to BioMed Central and take full advantage of:}

- Convenient online submission

- Thorough peer review

- No space constraints or color figure charges

- Immediate publication on acceptance

- Inclusion in PubMed, CAS, Scopus and Google Scholar

- Research which is freely available for redistribution 